\title{
A simple operative formula for ground level concentration from a point source
}

\author{
T. Tirabassi ${ }^{1}$, M. T. Vilhena ${ }^{2} \&$ D. Buske ${ }^{3}$ \\ ${ }^{1}$ Institute of Atmospheric Science and Climate (ISAC-CNR), \\ Bologna, Italy \\ ${ }^{2}$ Universidade Federal do Rio Grande do Sul (UFRGS), \\ Porto Alegre (RS), Brazil \\ ${ }^{3}$ Universidade Federal de Pelotas (UFPel), Pelotas (RS), Brazil
}

\begin{abstract}
Irreversible consequences of air pollution in the Atmospheric Boundary Layer (ABL) demand for increasing real time environmental monitoring and control as a routine instrument as well as for cases of environmental accidents or even catastrophes. In order to evaluate such scenarios one needs efficient procedures, which yield immediate results, for instance evaluating the ground level concentration of pollutants, and especially the maximum concentration and its position. The computational evaluation of numerical data of the concentration field or for a set of positions is an instant task using an analytical approach. So the Advection-Diffusion Equation is solved for a steady-state pollutant emission from a point-like source placed inside an unstable Atmospheric Boundary Layer. Then, an explicit approximate expression is provided for it, allowing an analytic simple expression for the position and value of the maximum.

Keywords: air pollution modelling, analytical solutions, advection-diffusion equation, maximum concentration, operative models, environmental management, integral transform.
\end{abstract}

\section{Introduction}

The prediction of pollutant dispersion in the atmosphere may be modeled by Eulerian and Lagrangian methods. The background of the Eulerian techniques is based upon the solution of the advection-diffusion equation. On the other hand, the framework of the Lagrangian models basically consists in the solution of the 
stochastic nonlinear Langevin equation. In this work we focus our attention on Eulerian models.

The analytical solution of the Advection-Diffusion Equation (ADE) has been performed following different approaches based on Gaussian and non-Gaussian solutions. Gaussian solutions represent a rather easy operative tool to be handled. Non-Gaussian analytical solutions represent a more realistic approach to represent atmospheric diffusion. Nonetheless using non-Gaussian approaches, solutions are much harder to be achieved, and often for rather simple parameterization profiles only. In Tirabassi [1] a short review in solving analytically the ADE is provided.

Due to the limitedness of generality and to the increasing development of Large Eddy Simulation (LES) models, analytical approaches to solve the ADE have been left aside. In this paper, a complete and coherent analytical solution of the ADE is presented. Such a solution is based on the Generalized Integral Laplace Transform Technique (GILTT) method [2]. The ability to handle the exact analytical solution allows upgrading the study of the concentration, nonetheless due to the non-explicit dependence on the set of variables defining the ABL scenario and the source features. In the GILTT solution the concentration is expressed through an infinite series expansion, so it could be useful to use an explicit analytical approximation of the Ground Level Concentration (GLC) and of its position when application purposes are required.

The paper focuses on GLC and the approximation of the GILTT solution. Such an explicit function allows the explicit determination of position and value of the maximum GLC respectively.

\section{The solution by GILTT}

The two-dimensional steady-state ADE for an emitting point-like source in a stationary ABL reads:

$$
u(z) \frac{\partial C(x, z)}{\partial x}=\frac{\partial}{\partial z}\left(k_{z}(z) \frac{\partial C(x, z)}{\partial x}\right),
$$

where along the $x$-direction the longitudinal diffusion term has been neglected in respect to the advection term. In the above equation $C(x, z)$ represents the cross-wind integrated three-dimensional time-independent concentration:

$$
C(x, z)=\int_{-\infty}^{+\infty} C(x, y, z) d y .
$$

The horizontal wind $u(z)$ is the horizontal mean wind and $k_{z}(z)$ is the vertical diffusivity; both are depending on the vertical coordinate $z$. The boundary conditions impose the flux to vanish at the extremes of the ABL $(z=0, h)$, and the source condition is set to represent the point-like source placed at the height $h_{S}$ above the ground level, namely: 


$$
u(z) C(0, z)=Q \delta\left(z-h_{S}\right),
$$

where $Q$ is the constant rate of emission and $\delta\left(z-h_{S}\right)$ is the Dirac $\delta$-function.

The GILTT technique provides a solution for eqn. (1) which is written in terms of a converging infinite series expansion [3]:

$$
C(x, z)=\sum_{i=0}^{\infty} \bar{c}_{i}(x) \phi_{i}(z)
$$

where $\phi_{i}(z)$ are the eigenfunctions of an auxiliary problem, i.e. solving the Sturm-Liouville equation, and $\bar{c}_{i}(x)$ are $x$-depending functions. As a consequence of convergence the series can be truncated at a certain number $N$ such that the rest $R_{N}(x, z)$ become negligible in respect of the partial sum. If one accepts an error not larger than $0.5 \%$ then $N=190$, as shown in [4].

\section{The turbulent parameterization}

The choice of the turbulent parameterization is set to account for the dynamics processes occurring in the ABL. In the further we restrict our discussion to simple vertical profiles of wind and eddy diffusivity, nevertheless still reasonably realistic, more specifically unstable regime. For an extension including stable regimens we refer to a future work. The choice of the vertical profile for the wind $u(z)$ is set to be following a power law [5]:

$$
\frac{u(z)}{u_{1}}=\left(\frac{z}{z_{1}}\right)^{\alpha},
$$

where $u_{1}$ is the mean wind velocity at the height $z_{1}$, while $\alpha$ is an exponent related to the turbulence intensity [6]. On the quantitative side, results will be provided setting $\alpha=0.1$, and the reference wind $u_{1}(0.01 h)=3 \mathrm{~ms}^{-1}$; these values are quite consistent with the whole range of unstable regimes pointed out by [7].

The vertical diffusivity parameterization is chosen according to Pleim and Chang [8], which for an unstable ABL it is given as:

$$
k_{z}(z)=k w_{*} z\left(1-\frac{z}{h}\right),
$$

where $h$ is the height of the ABL, $k$ is the von Karman constant which is set to 0.4 , and $w_{*}$ is the convective scaling parameter related to the Monin-Obukhov length $L_{M O}$ and the mechanical friction parameter $u_{*}$ as: 


$$
w_{*}=u_{*}\left(\frac{h}{L_{M O}}\right)^{1 / 3} .
$$

For convective scenarios, $L_{M O}$ is limited to values such that the relationship $\frac{h}{L_{M O}}<-10$ holds. Finally $u_{*}$ is determined as $[5,9]$ :

$$
u_{*}=u_{1} k\left(\ln \frac{z_{1}}{z_{0}}-\psi(\xi)\right)^{-1}
$$

where $z_{0}$ is the roughness $\left(10^{-5} h\right)$. For an unstable ABL $\psi$ defined as

$$
\psi(\xi) \cong \ln \left[\left(\frac{1+\xi^{2}}{2}\right)\left(\frac{1+\xi}{2}\right)^{2}\right]-2 \arctan \xi+\frac{\pi}{2}
$$

and

$$
\xi=\left(1-16 \frac{z_{1}}{L_{M O}}\right)^{\frac{1}{4}}
$$

The chosen profiles ensure simple functions and still rather realistic horizontal wind $u(z)$ and diffusivity $k_{z}(z)$ inside and both edges of the ABL.

\section{The approximate expression for ground level concentrations}

From the solution of the ADE the GLC is obtained after setting $z=0$ inside the solution $C(x, z)$. Results will be reported in terms of the dimensionless GLC as follows:

$$
C_{G L C}(x)=C(x, 0) \frac{<u>h}{Q},
$$

where $\langle u\rangle$ is the vertically averaged wind introduced in eqn. (5)

$$
<u=\frac{1}{h} \int_{0}^{h} u(z) d z
$$


If we consider the definition of $u$ profile in eqn. (5) we have $<u>=\frac{u_{1}}{\alpha+1}\left(h / z_{1}\right)^{\alpha}$.

Definition (11) has been introduced to obtain the unitary limit independent of a specific parameter choice

$$
\lim _{x \rightarrow \infty} C_{G L C}(x)=1,
$$

according to the theoretical expectation for the two-dimensional ADE solution.

It would be redundant to compare the GILTT results with experimental data as outcomes have been reported extensively in literature [10,11]. Rather the scope of this paper is to provide a simple explicit expression for the maximum GLC $\left(C_{M G L C}\left(x_{M}\right)\right)$ occurring at the horizontal distance $x_{M}$ as a function of the setting parameters for $\mathrm{ABL}$ scenario and source emission. As previously mentioned, in fact, although the sum (4) represents the exact solution of the ADE (1) except for a round-off error, the series expansion misses manifest dependencies on ABL parameters and source height. Then the core of the problem leads to investigate on the behaviour of the series (4) after setting $z=0$, and using the property of the Sturm-Liouville eigenfunctions for which $\phi_{i}(0)=1$ regardless the index $i$. An analysis of the behaviour and properties of the series (4) shall indicate how to synthesize the considerable expression into a more compact formula. The results based on such an approach are still profile depending and a general approximation is beyond the scope of the present work. Nevertheless, the choice of a profile depending approximation still maintains the advantage of simplicity and permits for a specific case to explore the functional behaviours of the main physical parameters that drive atmospheric diffusion. To this end we introduce empirical parameters which are determined by fit procedures to best reproduce the exact solution.

Based on these facts, and being in mind the Gaussian solution and the GLC obtained with power low profile of wind and eddy diffusivity, the dimensionless GLC defined in eqn. (11) can be approximated as follows:

$$
C_{G L C}(x)=\left[1+\left(\frac{\kappa h}{\lambda x}\right)^{c}\right]^{b} \exp \left[-\frac{\left(\pi h_{S}\right)^{1+2 b c}}{h(\lambda x)^{2 b c}}\right]
$$

Due to the negative values assumed by the Monin-Obukhov length, in the following it will be defined as the positive dimensionless parameter $\tilde{L}_{M O}=-L_{M O} / h$. Parameters $b, c, \kappa$ and $\lambda$ have been determined by least squares fittings procedures on eqn. (14) against the analytical solution and these are:

$$
b=\tilde{h}_{S}^{5 / 2}+.17,
$$




$$
\begin{gathered}
c=-5.48 \tilde{h}_{S}^{0.87}+4.73, \\
\kappa=\left(\frac{\alpha+1}{0.4277}\right)^{2.62} \tilde{h}_{S}^{0.41}, \\
\lambda=\left(0.35 u_{1}\right)^{-1}(\alpha+1)^{-1.3} w_{*} \tilde{h}_{S}^{.47} .
\end{gathered}
$$

where the variables with $\sim$ normalized with respect to the ABL height $h$ (e.g. $\left.\tilde{h}_{s}=h_{s} / h\right)$.

Equations (15)-(18) give the explicit dependency on the source height $h_{S}$, the wind parameters $\alpha$ (it compares in $k$ and $\lambda$ ), $u_{1}$ and the convection scaling parameter $\mathcal{w}_{*}$ (it compares in $\lambda$, see eqn. (18)) which is related to the MoninObukhov length $L_{M O}$ and the friction parameter $u_{*}$ by the relationship (7).

From the explicit approximation for $C_{G L C}(x)$ one may evaluate the position where the maximum for GLC occurs, in fact putting equal to 0 the derivative of eqn. (14) in respect to $x$ and with the assumption that:

$$
\left(\frac{\kappa h}{\lambda x}\right)^{c}>>1
$$

we have

$$
x_{M}^{2 b c}=2 \frac{\left(\pi h_{S}\right)^{1+2 b c}}{(h \lambda)^{2 b c}}
$$

Finally, putting $x_{M}$ in eqn. (14), the corresponding Maximum Ground Level Concentration $\left(C_{M G L C}\left(x_{M}\right)\right)$ is:

$$
C_{M G L C}\left(x_{M}\right)=\left[1+\left(\frac{2 \kappa}{\left(2 \pi \tilde{h}_{S}\right)^{1+\frac{1}{2 b c}}}\right)^{c}\right]^{b} e^{-\frac{1}{2}} .
$$

Two considerations are in order here. First, the expression for the position $x_{M}$ is valid provided that in the range of horizontal distances where a position $x_{M}$ occurs. Such approximation affects an error when high sources are concerned, indeed above $\tilde{h}_{S} \approx 0.35$, but high convection driven turbulence 
enforces condition (19). Second, because no maximum is reached for any $\tilde{h}_{S}>0.5$, the position of maxima in these cases has to be expected at $x \rightarrow \infty$ (due to the predominant weight of the exponential function compared to the first factor in eqn. (14)). For this reason the study of the maximum GLC will be limited to sources placed below the ABL centre level.

Figures 1 and 2 show plots of the maximum GLC $C_{M G L C}\left(x_{M}\right)$ and its position $x_{M}$ for several source height $\tilde{h}_{S}$ and for a selection of turbulence parameter $\tilde{L}_{M O}$. In the figures the GILTT results (points) are superimposed on the approximations (20) (dotted lines). Figure 1 depicts the position where the maximum occurs for low sources, where GILTT results (dotted lines) and our approximations (solid lines) show good matching regardless the turbulence regime. When higher sources are considered a difference shows up and increases as convective turbulence reduces its strength, this fact follows from the condition on (19). The turbulence dependency shows that for a fixed $\tilde{h}_{S}$ the strength of convection causes $x_{M}$ to get closer to the source. From the physics point of view this result agrees with the mixing effect of turbulence.

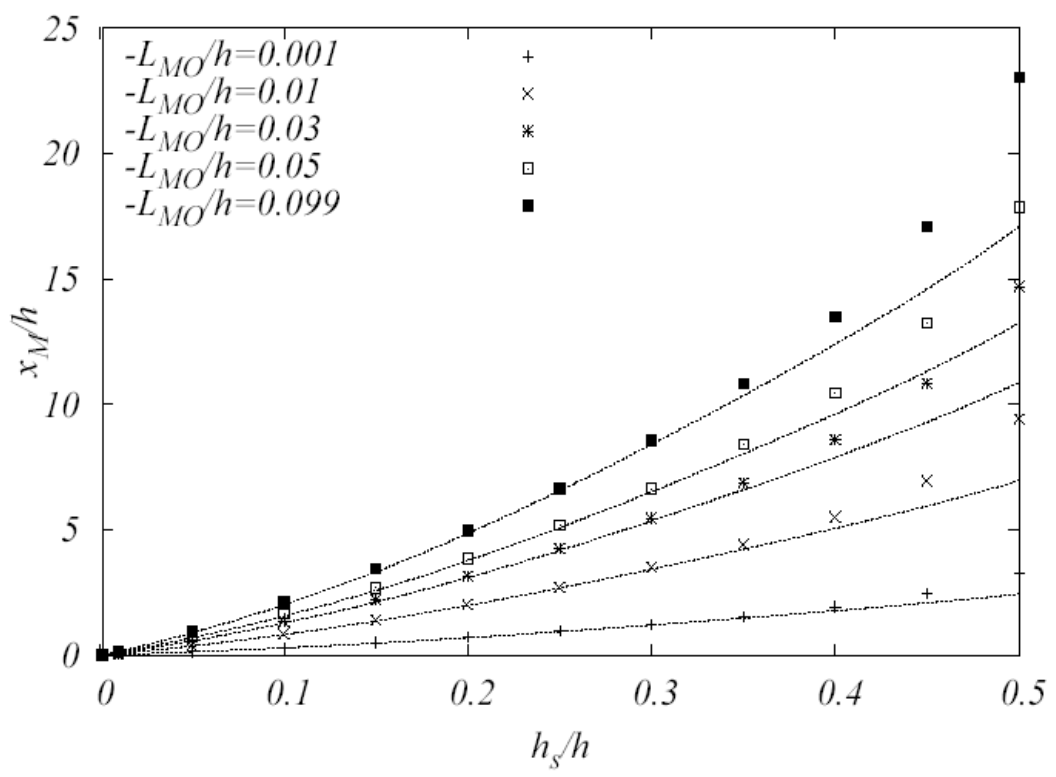

Figure 1: Position of the maximum GLC versus the source height $\tilde{h}_{S}=h_{S} / h$. Points refer to the GILTT results, dotted lines refer to eqn. (19).

A final remark should be made about fig. 2; both GILTT and expression (21) confirm that the maximum GLC value depends on the source height, regardless 
of the turbulence. Based on the expression (21) and the parameters definitions (15)-(16), for $b, c$ and $\kappa$, the leading term for the maximum GLC results:

$$
C_{M G L C}\left(x_{M}\right) \approx \tilde{h}_{S}^{-1},
$$

where the exponent -1 is a lower bound for the source term. We worth emphasizing that for a function given in (14), within the setting choice for the ABL parameter set, the maximum GLC depends only on the source height, regardless the Monin-Obukhov length. These results broaden the well known result obtained with the Gaussian approach for an unbounded ABL. Furthermore this agrees with the two-dimensional Gaussian result that the maximum for the GLC is:

$$
C_{M G L C}\left(x_{M}\right)=\left(\frac{2}{e \pi}\right)^{1 / 2}
$$

Note, that for the three-dimensional case this is no longer true. It is evident that any diffusive parameters do not enter and it confirms that turbulence has the only effect that determines the distance where maximum GLC occurs.

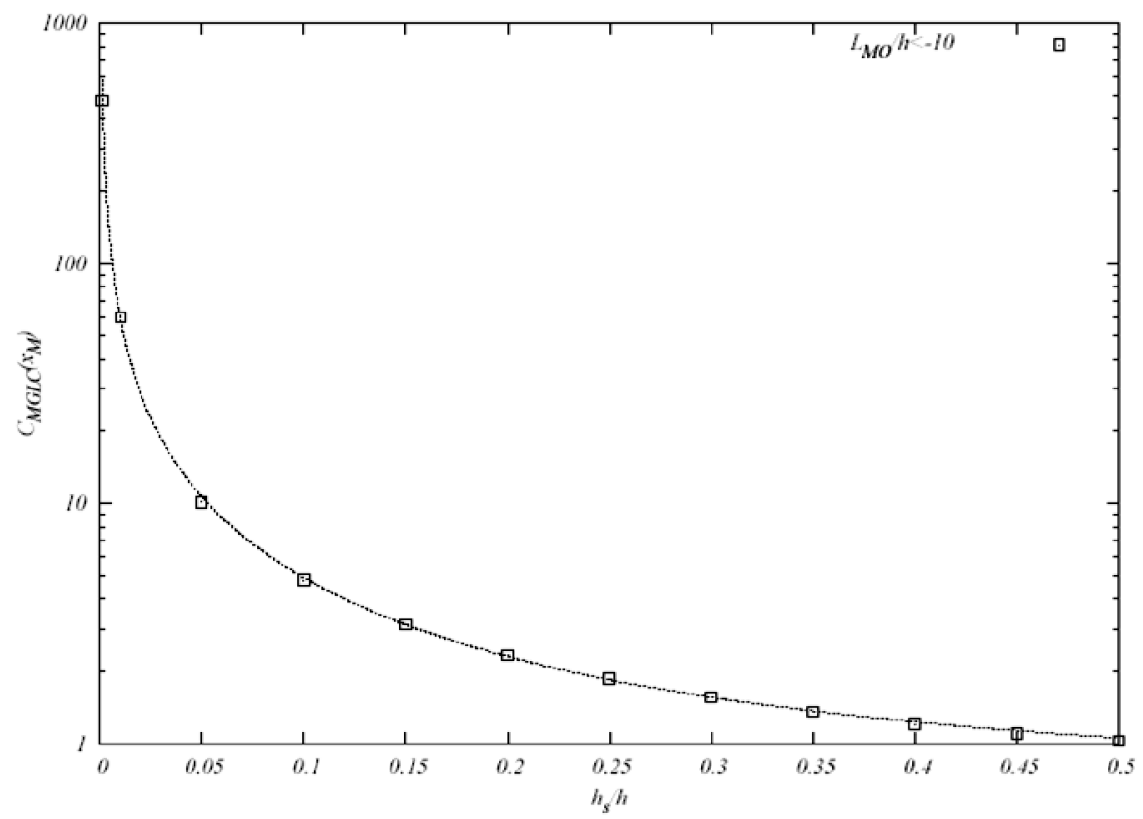

Figure 2: Value of the maximum GLC versus the source height $\tilde{h}_{S}=h_{S} / h$. Points refer to the GILTT results, dotted lines refer to eqn. (20). 


\section{Conclusions}

The results presented in this paper have shown the possibility to express the GLC from an emitting point-like source in a steady convective ABL, by a compact analytical expression. The function was determined analysing the behaviour of the series expansion provided by the GILTT solution, whose predictive power have been extensively demonstrated in the literature when applied to several experimental data sets.

Despite the simplifications due to restricting to only unstable ABL regimes, the analysis allows to understand to a high extent the form of the ground level concentration.

On the operative point of view, the expression (14) and its related features are useful as an additional tool for decisional as well as emergency responses.

\section{Acknowledgements}

The authors thank Brazilian CNPq and FAPERGS and Italian CNR for the partial financial support of this work.

\section{References}

[1] Tirabassi, T., Operational advanced air pollution modeling. PAGEOPH, 160 (1-2), pp. 5-16, 2003.

[2] Moreira, D.M., Vilhena, M.T., Buske, D. \& Tirabassi, T., The state-of-art of the GILTT method to simulate pollutant dispersion in the atmosphere, Atmos. Research, 92, pp. 1-17, 2009.

[3] Moreira, D.M., Vilhena, M.T., Tirabassi, T., Buske, D. \& Cotta, R.M., Near source atmospheric pollutant dispersion using the new GILTT method, Atmos. Environ., 39, pp. 6289-6294, 2005.

[4] Tirabassi, T., Tiesi, A., Buske, D. \& Vilhena, M.T., Some characteristics of a plume from a point source based on an analytical solution of the twodimensional advection-diffusion equation, Atmos. Environ., 43, pp. 22212227, 2009.

[5] Panofsky, H. A. \& Dutton, J. A., Atmospheric Turbulence, John Wiley \& Sons, New York, 1988.

[6] Irwin, J.S., A theoretical variation of the wind profile power-low exponent as a function of surface roughness and stability, Atmos. Environ., 13, pp. 191-194, 1979.

[7] Pasquill, F. \& Smith, F.B., Atmospheric Diffusion, John Wiley \& Sons, New York, 1984.

[8] Pleim, J. E. \& Chang, J. S., A nonlocal closure model for vertical mixing in the convective boundary-layer, Atmos. Environ. Part A-General Topics, 26, pp. 965-981, 1992.

[9] Zannetti, P., Air Pollution Modelling, Computational Mechanics Publications, Southampton, 1990. 
[10] Moreira, D.M., Vilhena, M.T., Buske, D. \& Tirabassi, T., The GILTT solution of the advection-diffusion equation for an inhomogeneous and nonstationary ABL, Atmos. Environ., 40, pp. 3186-3194, 2006.

[11] Buske, D., Vilhena, M.T., Moreira, D.M. \& Tirabassi, T., Simulation of pollutant dispersion for low wind conditions in stable and convective planetary boundary layer, Atmos. Environ., 41, pp. 5496-5501, 2007. 